\title{
On Reducing False Alarms in Multivariate Statistical Process Control
}

\author{
Tao Chen ${ }^{*}$ \\ School of Chemical and Biomedical Engineering, \\ Nanyang Technological University, Singapore 637459, Singapore
}

\begin{abstract}
The primary objective of this note is to reduce the false alarms in multivariate statistical process control (MSPC). The issue of false alarms is inherent within MSPC as a result of the definition of control limits. It has been observed that under normal operating conditions, the occurrence of "outof-control" data, i.e. false alarms, conforms to a Bernoulli distribution. Therefore this issue can be formally addressed by developing a Binomial distribution for the number of "out-of-control" data points within a given time window, and a second-level control limit can be established to reduce the false alarms. This statistical approach is further extended to consider the combination of multiple control charts. The proposed methodology is demonstrated through its application to the monitoring of a benchmark simulated chemical process, and it is observed to effectively reduce the false alarms whilst retaining the capability of detecting process faults.
\end{abstract}

Key words: Bernoulli distribution; Binomial distribution; false alarm; multivariate statistical process control; principal component analysis.

\section{Introduction}

Multivariate statistical process control (MSPC) is a critical approach to the monitoring of the performance of manufacturing processes for ensuring process safety and the delivery of high quality, consistent product. The basic procedure of MSPC consists of the following steps: (1) the development of a statistical model from historical data collected when the process runs under normal operating conditions; (2) the determination of control limits for the statistical model; and (3) the detection of process faults when on-line data exceeds the control limits, followed by the diagnoses of the cause of the faults. With the rapid development of automatic data collection systems, one of the primary concerns of MSPC is to effectively and efficiently utilize the large amount of data to characterize the process. This requirement has resulted in the application of multivariate statistical projection approaches, such as principal component analysis (PCA) (Wold and Geladi, 1987), partial least squares (PLS) (Geladi and Kowalski, 1986) and their non-linear variants (Choi et al., 2005; Zhao et al., 2006), to extract relevant process information, and to attain an enhanced understanding of process behaviour (Qin, 2003).

The emergence of a large variety of MSPC methods in the literature has required some criteria to assess and compare their performance. In analogous to general statistical detection problems, MSPC is concerned with two types of errors. Type I error, also termed false alarm, occurs when a normal data point is classified as faulty, whilst Type II error, or missing error, is the result of the failure to detect a non-conforming data point (i.e. process measurement at a time instance) (Montgomery, 2001). In the context of MSPC, an alternative definition of Type II error, the time delay between the onset of the fault and the fault being detected, is typically adopted to reflect the importance of an early indication of process anomaly.

\footnotetext{
${ }^{*}$ Corresponding author. E-mail: chentao@ntu.edu.sg; Tel.: +65 6513 8267; Fax: +65 67947553.
} 
The focus of this research note is to reduce the false alarms in MSPC. In Section 2 the motivation is presented through the discussion of the potential sources of false alarms in MSPC. Section 3 proposes a statistical approach to reducing the false alarms due to the random effects (defined subsequently). In Section 4 the proposed methodology is applied to the monitoring of a simulated continuous process with promising results. Finally Section 5 concludes this paper.

\section{MSPC and false alarms}

The basis of MSPC is to collect a set of historical data when the process is running under normal operating conditions (NOCs). For a continuous process NOCs can be the steady-state around which the process is operated, whilst for a batch process NOCs are described by the trajectory of the process measurements and manipulated variables that ensure the satisfactory product quality. Then multivariate statistical techniques, such as PCA, is applied to the historical data to extract the lowdimensional representation, and to develop the control limits for the monitoring statistics, the most widely used being the Hotelling's $T^{2}$ and squared prediction error ( $Q$ statistic). In the on-line monitoring stage, the monitoring statistics for the process measurement at each sampling time is calculated based on the PCA model. If the statistics exceed the control limit, then the data point (and thus the process) is detected as "out-of-control".

Once an onset of fault is detected, the next step is to identify the source or root cause of the process fault. The task of fault diagnosis can be achieved in a number of ways, including the contribution analysis that aims to identify the variables that contribute the most to the fault, statistical pattern recognition techniques and expert systems (Qin, 2003). The primary objective of this paper is to reduce the false alarms in process monitoring through MSPC, and thus the discussion on fault diagnosis is outwith the scope of this paper.

The occurrence of false alarms may be attributed to a number of causes that are grouped into two categories in this study, including systematic model deficiency and random effects. Systematic model deficiency may be due to the mismatch between the statistical model and the process being monitored. A typical example in chemical processes is that a PCA model, which was developed using the historical data under one operating mode, is applied to monitor the process running under another operating mode. This issue can be addressed through the update of the model to incorporate new normal operating conditions, such as using recursive PCA (Li et al., 2000). Model deficiency may also originate from the violation of model assumptions. For example, the application of PCA is based the assumption that the process data is independent and identically Gaussian distributed. However in practice the process variables tend to be auto-correlated, necessitating time-series modelling approaches to reduce the resultant false alarms (Alabi et al., 2005; Kruger et al., 2004; Xie et al., 2006). Furthermore, in some situations the multivariate Gaussian distribution may be an inadequate approximation to the real process variables, and thus more advanced semi-parametric and non-parametric distributions are required to characterize the process normal behaviour accurately, including kernel density estimation (Martin and Morris, 1996), wavelet based density estimation (Safavi et al., 1997), and more recently the Gaussian mixture model (Chen and Sun, 2009; Choi et al., 2004; Thissen et al., 2005).

In addition to model deficiency, there are some random effects which may cause false alarms. Some on-line fluctuation of the process being monitored is inevitable, and this random fluctuation can result in the process variables being deviated from their nominal values, whilst the process is running normally according to safety and product quality requirements. More importantly, randomly induced false alarms are inherent within MSPC because of the definition of the control limits. For example, the $99 \%$ control limit, also known as "action limit", states that statistically $1 \%$ of normal operating data will fall outside this limit, materializing in false alarms. This issue is more 
serious when the $95 \%$ control limit, known as "warning limit", is used since statistically $5 \%$ of the normal data points will be incorrectly identified as faulty. The presence of false alarms is one of the major reasons that the plant operators are reluctant to employ MSPC technology for process fault detection and diagnose. In industrial practice, a heuristic rule, though lacking theoretic foundations, has been suggested to signal the onset of the process fault when (1) two data points (within a specified time window) lie outside the warning limit; or (2) one data point exceeds the action limit (Martin and Morris, 1996).

The primary goal of this paper is to suppress the randomly induced false alarms through the development of a statistical approach. It is assumed that the systematic model deficiency can be addressed using the methods reviewed previously, and thus will be not considered in this study.

\section{Reducing randomly induced false alarms}

This section presents a statistical approach to reducing the randomly induced false alarms. This approach was originally proposed in the literature of time-series outlier detection to differentiate the presence of randomly induced outliers from the onset of systematic process change (Abraham and Chuang, 1993; Chen et al., 2008; Singhal and Seborg, 2000).

\subsection{The statistical framework}

In general we consider a $100 \alpha \%$ control limit that is used for process fault detection. Under normal operating conditions, the probability of the occurrence of "out-of-control" data points, i.e. false alarms, is equal to $1-\alpha$. More formally let $c_{i}$ be an indicator variable to denote whether the process at time step $i$ has been identified as faulty. Hence $c_{i}$ is a Bernoulli random variable with the probability:

$$
p\left(c_{i}\right)=\left\{\begin{array}{ccl}
1-\alpha & c_{i}=1 & \text { (faulty) } \\
\alpha & c_{i}=0 & \text { (normal) }
\end{array}\right.
$$

We further consider a sequence of such Bernoulli random variables within a time window of size $n$ : $c_{i-n+1}, \cdots, c_{i}$. According to probability theory (Papoulis, 1984), if $c_{i-n+1}, \cdots, c_{i}$ are independent and identically distributed according to Eq. (1), then the sum, $m_{i}=\sum_{t=1}^{n} c_{i-n+t}$ is a Binomial random variable with the following probability density function:

$$
p\left(m_{i} ; \alpha, n\right)=\left(\begin{array}{c}
n \\
m_{i}
\end{array}\right)(1-\alpha)^{m_{i}} \alpha^{n-m_{i}}
$$

where $m_{i}$ is also equal to the number of $c_{i}$ 's that are equal to one. The assumption of independent $c_{i}$ 's is in accordance with the assumption in MSPC that process data is independent. If severe serial correlation is present, dynamic techniques should be utilized to model the data, as discussed in Section 2.

In the context of process monitoring, Eq. (2) amounts to assuming that at time step $i$, the number of data points being identified as faulty in the time window $(i-n+1, \cdots, i)$, is a Binomial random 
variable as given by Eq. (2). Therefore a $100 \beta \%$ control limit can be established for $m_{i}$, the limit denoting the maximum number of out-of-control data points allowed, $m_{100 \beta}$, in a time window of size $n$ (Abraham and Chuang, 1993; Chen et al., 2008; Singhal and Seborg, 2000):

$m_{100 \beta \%}=\max m, \quad$ such that $: F(m ; \alpha, n)=\sum_{j=0}^{m}\left(\begin{array}{c}n \\ j\end{array}\right)(1-\alpha)^{j} \alpha^{n-j}<\beta$

where $m_{100 \beta \%}$ is obtained by calculating the cumulative distribution function (cdf), $F(m ; \alpha, n)$, for $m$, and then selecting the maximum value of $m$ that satisfies Eq. (3). Typically the randomly induced out-of-control data will fall within this limit, whereas the limit being exceeded will indicate the onset of a process fault requiring operators' attention. By utilizing the property of the Bernoulli and Binomial distributions in conjunction with MSPC, the proposed method will be able to reduce the false alarms induced by random effects, whilst attaining fault detection capability. For the reason of clarity, $100 \alpha \%$ will be referred to as "MSPC control limit" and $m_{100 \beta \%}$ as "second-level control limit" onwards.

In summary the proposed approach executes as follows:

1. At time step $i$, apply PCA to the process measurement and calculate the $T^{2}$ statistic. For clarity of presentation we consider a single monitoring statistic. The extension to multiple statistics is discussed in the next sub-section.

2. If the $100 \alpha \%$ control limit for $T^{2}$ is violated, then count the number of data points that violated the $100 \alpha \%$ limit in the past $n$ time steps (including current time);

- If this number exceeds the second-level control limit $m_{100 \beta \%}$, then an onset of process fault is detected;

- Else the process is classified as under normal operating conditions.

Else the process is classified as under normal operating conditions.

To implement the second-level control limit based on the Binomial distribution, there are several parameters to be determined, including $\beta$ and $n$. Following the suggestions in the literature of outlier detection (Abraham and Chuang, 1993; Chen et al., 2008; Singhal and Seborg, 2000), the second-level control limit, $\beta$, is typically taken as 0.99 . The selection of the window size $n$ must ensure that there is at least one out-of-control data point allowed in this window, i.e. $m_{100 \beta \%} \geq 1$, since $m_{100 \beta \%}=0$ is equivalent to using the conventional MSPC. On the other side, the window size should be relatively small to keep a small value of $m_{100 \beta \%}$, and hence to attain a sufficient sensitivity to the real process faults. According to Eq. (3), $m_{100 \beta \%}$ is also dependent on the value of the MSPC control limit, $100 \alpha \%$. Table 1 gives the value of $m_{99 \%}$ at different window size and $\alpha$, where it appears that a window size of 20 is a good choice to satisfy these requirements for both 95\% and 99\% MSPC control limit, and this value will be used in this study. Nevertheless, the choice of a proper window size is rather subjective. Alternatively, if available, historical faulty data may be used to evaluate the impact of window size on the sensitivity of fault detection, and this information is highly valuable to select a sensible window size.

(Table 1 about here) 


\subsection{Extension to multiple control charts}

The above strategy can be extended to consider multiple control charts simultaneously, such as the combination of charts for $T^{2}$ and $Q$ statistics that are widely used in conjunction with PCA and PLS models. When multiple MSPC control limits, corresponding to multiple charts, are utilized, the process can be identified as faulty when any of the control limits is exceeded (Chen and Sun, 2009), since the control charts typically characterize different aspects of the process and thus are complementary. For example, in the application of PCA model, $T^{2}$ monitors the covariance structure of process variables, whereas the $Q$ statistic ensures a small magnitude of prediction errors. To derive the second-level control limits for the combination of $T^{2}$ and $Q$ statistics, two events are defined:

- $\quad$ Event $A$ : The control limit of $T^{2}$ is exceeded;

- Event $B$ : The control limit of the $Q$ statistic is exceeded.

Therefore the probability of either of the two events happens is:

$$
P(A \cup B)=P(A)+P(B)-P(A \cap B)
$$

By assuming the MSPC control limits are the same for both statistics $(P(A)=P(B)=100 \alpha \%)$, and the two events are independent $\left(P(A \cap B)=P(A) P(B)=\alpha^{2}\right)$, Eq. (4) can be calculated analytically as: $P(A \cup B)=2 \alpha-\alpha^{2}$. Alternatively $P(A \cup B)$ can be calculated empirically as follows. We can first calculate $T^{2}$ and $Q$ statistics of the nominal process data that are used for the development of the PCA model, and then set $P(A \cup B)$ as the frequency of either Event $A$ or $B$ happens within the nominal data set. In this study, simulation results have found that the two ways of calculating $P(A \cup B)$ give similar values (not reported), and thus the independence assumption is reasonable. In the case study presented in Section 4 , the analytical calculation, i.e. $P(A \cup B)=2 \alpha-\alpha^{2}$, is adopted.

In analogous to the discussion in Section 3.1, the joint event, $A \cup B$, conforms to a Bernoulli distribution with the parameter $\alpha$ in Eq. (1) being replaced by $2 \alpha-\alpha^{2}$. Therefore the secondlevel control limit can be constructed for multiple charts as presented in Section 3.1. The extension of this strategy to more than two control charts is straightforward and is neglected here. Table 2 gives the value of $m_{99 \%}$ at different window size and $\alpha$ for two control charts, where it appears that a reasonable choice of window size can be: $n=15$ when $\alpha=0.95$, and $n=20$ when $\alpha=0.99$.

(Table 2 about here)

\section{Case study}

\subsection{The Tennessee Eastman Process}

The Tennessee Eastman process (Downs and Vogel, 1993) was provided as a benchmark for testing new methodologies in advanced process control and process performance monitoring. It has been implemented in Matlab Simulink environment and used extensively in the literature of MSPC (Kruger et al., 2004). The process comprises a set of unit operations (reactor/separator/stripper/compressor) with two simultaneous exothermic reactions and two byproduct reactions. In this study, the simulation software is run under the base mode (mode 1) with a 
decentralized control strategy (Ricker, 1996). The process has 12 manipulated variables and 41 measurements. However a number of the quality measurements, such as product concentration, are only available infrequently in industrial scale plant and hence were removed from the analysis. Thus the final data set that was used to build the model comprised 22 measurements, plus 12 manipulated variables. The details of these 34 variables can be found in (Downs and Vogel, 1993). The sampling interval was $0.02 \mathrm{~h}$.

To evaluate the effectiveness of the proposed approach, five faulty types are considered, as listed in Table 3. From previous analyses that have been reported in the literature, it is acknowledged that fault "IDV(1)" and "IDV(4)" results in a direct step change in process measurements and thus are relatively easy to detect. Fault "IDV(14)" is related to a sticky valve for reactor cooling water, and the disturbance is expected to be reflected in the reactor temperature measurement. In contrast fault "IDV(10)" is more subtle as it disturbs the reactant feed temperature which was not directly measured. Finally "IDV $(12+15)$ " is the most complicated, as it reflects the simultaneous onset of two faults, a disturbance in an unmeasured variable and a device fault, and thus it is challenging to detect. For each fault scenario the process was initially run for 20 hours under normal operating conditions, giving 1000 data points, each being a vector of 34 dimensions. The first 500 data were selected to define the nominal operating region, and the remaining 500 data points were reserved to assess the false alarm rate. The process was then run under the conditions that simulated faulty behaviour for a further 4 hours, giving 200 faulty data points. Note the benchmark simulation software encapsulates the details of implementation and it automatically determines the magnitude of measurement noise and faulty signal according to the typical process operations (Downs and Vogel, 1993).

(Table 3 about here)

\subsection{Results and Discussions}

The data set is pre-processed to have zero mean and unit standard deviation at each variable. Then PCA was performed on the pre-processed nominal data set (500 data points), and the number of principal components retained is determined using five-fold cross-validation. We first investigate the monitoring charts for fault "IDV $(12+15)$ " as shown in Figure 1, where the fault was introduced at time point $20 \mathrm{~h}$ and the $95 \% \mathrm{MSPC}$ control limit was utilized. It is clearly seen that prior to the introduction of the process fault, there are a significant number of false alarms for both $T^{2}$ and $Q$ statistics, with false alarm rates being $2.8 \%$ and $8.6 \%$ respectively. Typically a small false alarm rate implies that the control chart tends to be less sensitive to process fault, and it may result in large detection delay. This phenomenon has been observed in this example, where the detection delays for $T^{2}$ and $Q$ statistics are $0.30 \mathrm{~h}$ and $0.12 \mathrm{~h}$ respectively, i.e. $T^{2}$ gives fewer false alarms but is less sensitive to the process fault than the $Q$ statistic does.

(Figure 1 about here)

Figure 2 depicts the monitoring charts for fault "IDV(12+15)" using the second-level control limit for the number of out-of-control data points within a window of 20 time steps as described in Section 3. Compared with Figure 1, the salient feature of Figure 2 is that the number of false alarms has been significantly reduced for both $T^{2}$ and $Q$ control charts: the corresponding false alarm rates have dropped to $0.4 \%$ (from $2.8 \%$ ) and $0.8 \%$ (from 8.6\%). Furthermore, the reduction of false alarms has not significantly sacrificed the detection delay, where the delay is increased from $0.30 \mathrm{~h}$ to $0.36 \mathrm{~h}$ for $T^{2}$, but not changed (both are $0.12 \mathrm{~h}$ ) for the $Q$ statistic.

(Figure 2 about here) 
Figure 3 illustrates the use of a second-level control limit to combine $T^{2}$ and $Q$ control charts for fault "IDV $(12+15)$ ", where again most of the false alarms have been eliminated without significant impact on the detection delay. The false alarm rate has been reduced from $10.6 \%$ to $0.4 \%$ whilst the detection delay is increased from $0.12 \mathrm{~h}$ to $0.14 \mathrm{~h}$. The relatively high false alarm rate associated with multiple charts is because the process is identified as abnormal when either chart indicates so. Therefore the proposed approach is especially applicable in the situation of multiple charts that are widely used in the implementation of MSPC techniques.

\section{(Figure 3 about here)}

Table 4 and 5 summarize the monitoring results for all the five faulty scenarios using $95 \%$ and $99 \%$ MSPC control limits, respectively. In all cases the proposed second level control limit effectively suppresses the false alarms. When using the 95\% MSPC control limit, on average the false alarm rates are decreased from $8.7 \%$ to $2.0 \%$ with the detection delay being marginally increased from $0.17 \mathrm{~h}$ to $0.20 \mathrm{~h}$. It could be argued that false alarms can be suppressed by simply choosing the $99 \%$ MSPC control limit. However in practice, given a set of nominal process data, it is more reliable to estimate the $95 \%$ control limit than the $99 \%$ limit, since the estimation of a "rare event" such as the 99\% control limit is highly sensitive to small change in the data (Rychlik and Ryden, 2006). In addition, the use of $99 \%$ control limit could result in significantly longer detection delay. For example the detection delay for fault "IDV $(12+15)$ ", using combined $T^{2}$ and Q statistics, is $0.12 \mathrm{~h}$ for $95 \%$ limit but $0.30 \mathrm{~h}$ for $99 \%$ limit. Therefore the choice of the two control limits is dependent on practical requirements and they are typically considered together to keep a balance between robustness (fewer false alarms) and sensitivity (shorter detection delay). However even if 99\% MSPC control limit is desirable and utilized, Table 5 shows that by utilizing the proposed approach, the false alarm rates can still be reduced from $2.2 \%$ to $0.8 \%$ on average, with the detection delay being slightly increased from $0.23 \mathrm{~h}$ to $0.24 \mathrm{~h}$.

\section{Conclusions}

This paper proposes a statistical approach to reducing the false alarms in multivariate statistical process control. While there are many potential causes of false alarms, this study is focused on those induced by random effects. The central component of this statistical approach is to construct a second-level control limit that is based on the known properties of Bernoulli and Binomial distributions. The proposed methodology is simple in its concept, straightforward to implement, effective in reducing the false alarms, and capable of retaining the fault detection power of the original MSPC methods. Besides the conventional PCA based method, this statistical framework is generally applicable to other process monitoring techniques, including the dynamic and mixture models reviewed in Section 2.

In practice, a potential limitation of the proposed methodology is that the use of a time window may result in significant detection delay for processes with very low rate of data collecting, such as some batch fermentation processes. For example, if the sampling rate of the process is one hour, a detection delay of one data point (corresponding to $0.02 \mathrm{~h}$ in the case study in Section 4) turns out to be $1 \mathrm{~h}$, and thus is highly undesirable. Alternative approaches for these "slow" processes should be utilized and they are under investigation.

\section{References}

Abraham, B. and Chuang, A., 1993, Expectation-Maximization algorithms and the estimation of time series models in the presence of outliers, J. Time Series Anal., 14: 221-234. 
Alabi, S.I., Morris, A.J. and Martin E.B., 2005, On-Line dynamic process monitoring using wavelet-based generic dissimilarity measure, Chem. Eng. Res. Des., 83: 698-705.

Chen, T. and Sun, Y., 2009, Probabilistic contribution analysis for statistical process monitoring: a missing variable approach, Contr. Eng. Pract., 17: 469-477.

Chen, T., Morris, J. and Martin, E., 2008, Dynamic data rectification using particle filters, Comput. Chem. Eng., 32: 451-462.

Choi, S. W., Park, J. H. and Lee, I.-B., 2004, Process monitoring using a Gaussian mixture model via principal component analysis and discriminant analysis, Comput. Chem. Eng., 28: 13771387.

Choi, S. W., Lee, C., Lee, J.-M. and Lee, I.-B., 2005, Fault detection and identification of nonlinear processes based on kernel PCA, Chemometr. Intell. Lab. Syst., 75: 55-67.

Downs, J. J. and Vogel, E. F., 1993, A plant-wide industrial process control problem. Comput. Chem. Eng., 17: 245-255.

Geladi, P. and Kowalski, B., 1986, Partial least-squares regression: a tutorial, Anal. Chim. Acta, 185: $1-17$.

Kruger, U., Zhou, Y. and Irwin, G. W., 2004, Improved principal component monitoring of largescale processes, J. Process Contr., 14: 879-888.

Li, W., Yue, H. H., Valle-Cervantes, S. and Qin, S. J., 2000, Recursive PCA for adaptive process monitoring, J. Process Contr., 10: 471-486.

Martin, E. B. and Morris, A. J., 1996, Non-parametric confidence bounds for process performance monitoring charts, J. Process Contr., 6: 349-358.

Montgomery, D. C., 2001, Introduction to Statistical Quality Control (4th Edition); Wiley: New York.

Papoulis, A., 1984, Probability, Random Variables, and Stochastic Processes (2nd Edition). McGraw-Hill: New York.

Qin, S. J., 2003, Statistical process monitoring: basics and beyond, J. Chemometr., 17: 480-502.

Ricker, N. L., 1996, Decentralized control of the Tennessee Eastman challenge process, J. Process Contr., 6: 205-221.

Rychlik, I. and Ryden, J., 2006, Probability and Risk Analysis - An Introduction for Engineers. Springer.

Safavi, A. A., Chen, J. and Romagnoli, J. A., 1997, Wavelet-based density estimation and application to process monitoring, AIChE J., 43: 1227-1241.

Singhal, A. and Seborg, D. E., 2000, Dynamic data rectification using the expectation maximization algorithm, AIChE J., 46: 1556-1565.

Thissen, U., Swierenga, H., de Weijer, A. P., Wehrens, R., Melssen, W. J. and Buydens, L. M. C., 2005, Multivariate statistical process control using mixture modelling, J Chemometr., 19: 23-31.

Wold, S. K. and Geladi P., 1987, Principal component analysis, Chemometr. Intell. Lab. Syst., 2: 37-52.

Xie, L., Kruger, U., Lieftucht, D., Littler, T., Chen, Q. and Wang, S.-Q., 2006, Statistical monitoring of dynamic multivariate processes - Part 1. Modeling autocorrelation and crosscorrelation. Ind. Eng. Chem. Res., 45: 1659-1676.

Zhao, S. J., Zhang, J., Xu, Y. M. and Xiong, Z. H., 2006, A nonlinear projection to latent structures method and its applications, Ind. Eng. Chem. Res., 45: 3843-3852. 


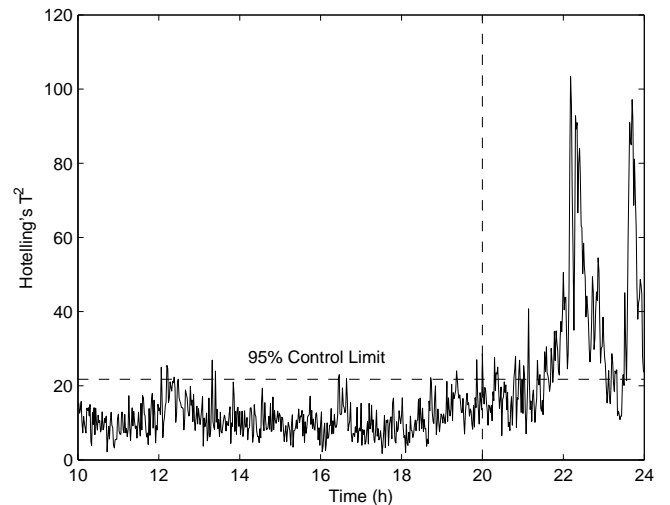

(a)

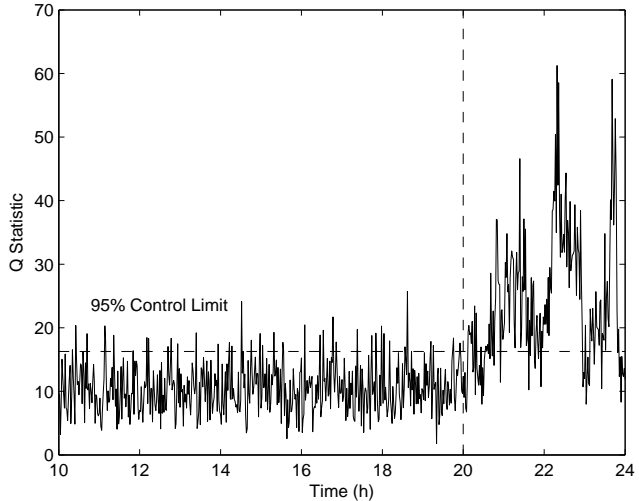

(b)

Figure 1: Process monitoring charts for (a) $T^{2}$ and (b) Q statistics, where the fault was introduced at $20 \mathrm{~h}$.

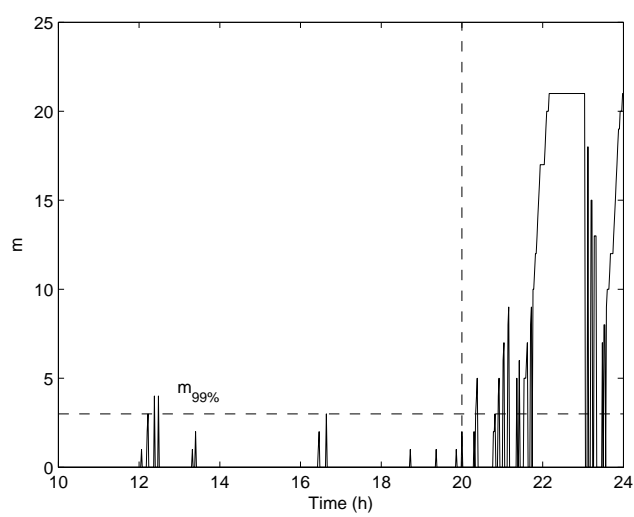

(a)

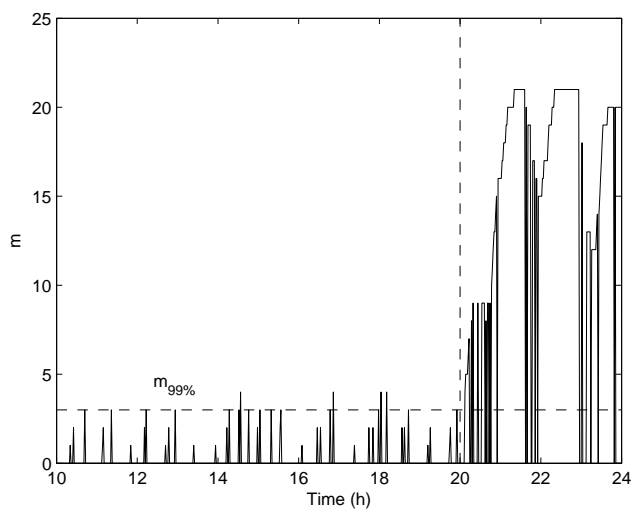

(b)

Figure 2: Process monitoring using the second-level control limit for : (a) $T^{2}$ and (b) Q statistics, where the fault was introduced at $20 \mathrm{~h}$.

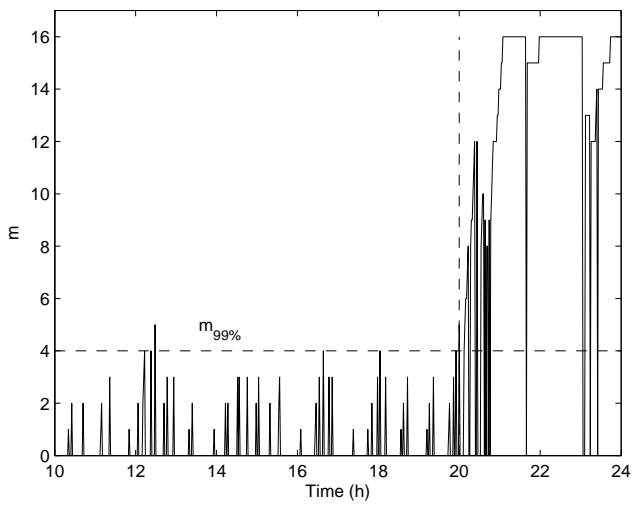

Figure 3: Process monitoring using the second-level control limit for the combination of $T^{2}$ and Q statistics, where the fault was introduced at $20 \mathrm{~h}$. 
Table 1: The second-level control limit, $m_{99 \%}$, at different window size ( $\left.n\right)$ and the MSPC control limit $(100 \alpha \%)$.

\begin{tabular}{lllllll}
\hline $100 \alpha \%$ & $n=5$ & 10 & 15 & 20 & 25 & 30 \\
\hline $95 \%$ & 1 & 2 & 2 & $\mathbf{3}$ & 3 & 4 \\
$99 \%$ & 0 & 0 & 0 & $\mathbf{1}$ & 1 & 1 \\
\hline
\end{tabular}

Table 2: The second-level control limit, $m_{99 \%}$, at different window size $(n)$ and the MSPC control limit $(100 \alpha \%)$ for the combination of two control charts.

\begin{tabular}{lllllll}
\hline $100 \alpha \%$ & $n=5$ & 10 & 15 & 20 & 25 & 30 \\
\hline $95 \%$ & 1 & 3 & $\mathbf{4}$ & 4 & 5 & 6 \\
$99 \%$ & 0 & 0 & 0 & $\mathbf{1}$ & 2 & 2 \\
\hline
\end{tabular}

Table 3: Process faults.

\begin{tabular}{cc}
\hline Case & Disturbance \\
\hline IDV(1) & A/C feed ratio (step change) \\
IDV(4) & Reactor cooling water inlet temperature (step change) \\
IDV(10) & C feed temperature (random variation) \\
IDV(14) & Reactor cooling water valve (sticking) \\
IDV(12+15) & Condenser cooling water inlet temperature (random variation) \\
& and condenser cooling water valve (sticking) \\
\hline
\end{tabular}


Table 4: Process monitoring results by using 95\% MSPC control limit (i.e. $100 \alpha \%=95 \%$ ).

\begin{tabular}{cccccc}
\hline \multirow{2}{*}{ Case } & & \multicolumn{2}{c}{ False Alarm (\%) } & \multicolumn{2}{c}{ Detection Delay (h) } \\
\cline { 2 - 6 } & $T^{2}$ & MSPC & MSPC + 2nd level & MSPC & MSPC + 2nd level \\
\hline IDV(1) & $Q$ & 9.0 & 0.0 & 0.10 & 0.14 \\
& $T^{2}+Q$ & 11.0 & 2.4 & 0.06 & 0.10 \\
IDV(4) & $T^{2}$ & 5.6 & 0.8 & 0.06 & 0.06 \\
& $Q$ & 12.2 & 3.4 & 0.04 & 0.04 \\
& $T^{2}+Q$ & 16.8 & 5.4 & 0.04 & 0.06 \\
IDV(10) & $T^{2}$ & 2.6 & 7.8 & 0.04 & 0.04 \\
& $Q$ & 10.0 & 1.6 & 0.56 & 0.66 \\
IDV(14) & $T^{2}+Q$ & 12.4 & 2.4 & 0.46 & 0.52 \\
& $T^{2}$ & 4.4 & 1.0 & 0.46 & 0.52 \\
& $Q$ & 9.4 & 0.2 & 0.08 & 0.14 \\
IDV(12+15) & $T^{2}+Q$ & 13.2 & 2.2 & 0.06 & 0.06 \\
& $T^{2}$ & 2.8 & 1.0 & 0.06 & 0.08 \\
& $Q$ & 8.6 & 0.8 & 0.30 & 0.36 \\
Average & $T^{2}+Q$ & 10.6 & 0.4 & 0.12 & 0.12 \\
& & $\mathbf{8 . 7}$ & 0.4 & 0.12 & 0.14 \\
& & & $\mathbf{2 . 0}$ & $\mathbf{0 . 1 7}$ & $\mathbf{0 . 2 0}$ \\
\hline
\end{tabular}

Table 5: Process monitoring results by using 99\% MSPC control limit (i.e. $100 \alpha \%=99 \%$ ).

\begin{tabular}{|c|c|c|c|c|c|}
\hline \multirow{2}{*}{ Case } & & \multicolumn{2}{|c|}{ False Alarm (\%) } & \multicolumn{2}{|c|}{ Detection Delay (h) } \\
\hline & & MSPC & MSPC + 2nd level & MSPC & MSPC + 2nd level \\
\hline \multirow[t]{3}{*}{ IDV(1) } & $T^{2}$ & 0.4 & 0.0 & 0.10 & 0.10 \\
\hline & $Q$ & 2.0 & 0.6 & 0.08 & 0.10 \\
\hline & $T^{2}+Q$ & 2.2 & 0.8 & 0.08 & 0.10 \\
\hline \multirow[t]{3}{*}{$\operatorname{IDV}(4)$} & $T^{2}$ & 1.6 & 1.0 & 0.04 & 0.04 \\
\hline & $Q$ & 3.6 & 1.4 & 0.04 & 0.04 \\
\hline & $T^{2}+Q$ & 5.0 & 2.2 & 0.04 & 0.04 \\
\hline \multirow[t]{3}{*}{ IDV(10) } & $T^{2}$ & 1.2 & 0.2 & 0.72 & 0.74 \\
\hline & $Q$ & 3.0 & 1.2 & 0.46 & 0.48 \\
\hline & $T^{2}+Q$ & 3.8 & 1.8 & 0.46 & 0.48 \\
\hline \multirow[t]{3}{*}{ IDV(14) } & $T^{2}$ & 0.2 & 0.0 & 0.10 & 0.12 \\
\hline & $Q$ & 3.2 & 1.6 & 0.06 & 0.08 \\
\hline & $T^{2}+Q$ & 3.4 & 1.8 & 0.06 & 0.08 \\
\hline \multirow[t]{3}{*}{$\operatorname{IDV}(12+15)$} & $T^{2}$ & 1.2 & 0.0 & 0.30 & 0.30 \\
\hline & $Q$ & 1.0 & 0.0 & 0.54 & 0.62 \\
\hline & $T^{2}+Q$ & 1.2 & 0.0 & 0.30 & 0.30 \\
\hline Average & & 2.2 & 0.8 & 0.23 & 0.24 \\
\hline
\end{tabular}

